\title{
Parry- Romberg Syndrome: A Case Report
}

\section{Devani P, Hiralal, Thakral A}

Department of Radiology, SGPGIMS, Lucknow, India

\begin{abstract}
The purpose of this report is to present radiological features of a rare entity called ParryRomberg syndrome (also known as Progressive hemifacial atrophy). The authors report one rare case of a 18 year old female patient with Parry-Romberg syndrome, accompanied by a brief review of literature and various radiological features of this entity. Final diagnosis of a Parry-Romberg syndrome was made on clinical and radiological grounds. Radiologists should be familiar with various radiographic, CT and MRI findings observed in this disorder.
\end{abstract}

Keywords: Computed Tomography, Parry-Romberg syndrome, Progressive Hemifacial Atrophy

\section{Introduction}

Parry-Romberg syndrome, is also called progressive facial hemiatrophy. It is a rare idiopathic neurocutaneous syndrome, probably acquired autoimmune disorder that causes atrophy of the soft tissues (usually starts with subcutaneous fat) and sometimes involves the bones on one half of the face without facial muscle weakness. ${ }^{1,2,3}$ At times atrophy extends to the ipsilateral limbs. It is associated with various ophthalmological and neurological complications. It bears many similarities with scleroderma. ${ }^{1}$

\section{Case Report}

A 18-year-old girl presented with gradually

Corrospondence to: Dr Hira Lal,
Department of Radiology, SGPGIMS, Lucknow, U.P (State) - 226014, India Email Id- hiralal2007@yahoo.co.in progressive right facial asymmetry and a small area of depression on right head.

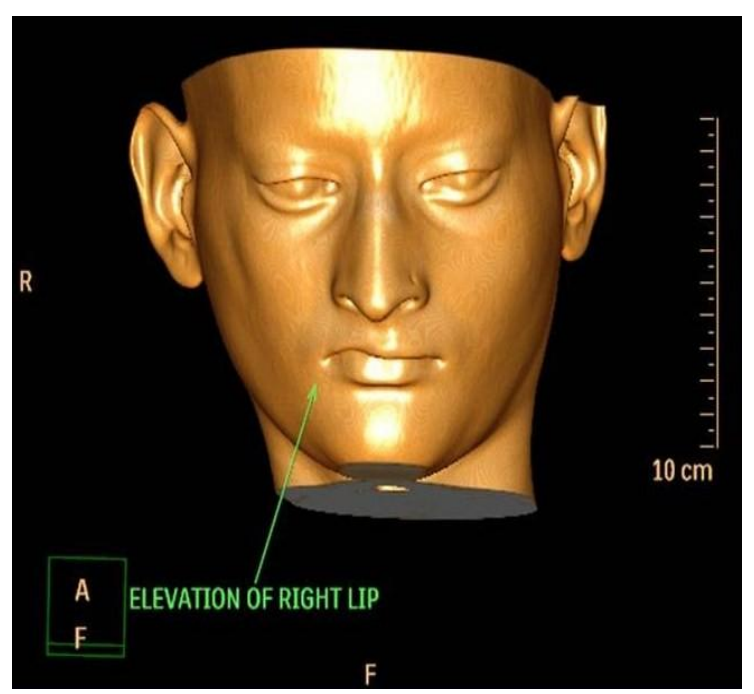

Fig 1: Volume Rendered Image shows overall facial asymmetry with elevation of right lip and depression of right cheek.

Physical examination further revealed elevation of the right lip and mild deviation of nose on right side alongwith tightening of her skin and mild depression in scalp on 
right side. On clinical examination, was no evidence of facial nerve palsy or trigeminal dysfunction. Mastication was normal. She had no other underlying illness.

Unenhanced CT of the face was performed, and images were viewed with bone and softtissue window settings. Volume-rendered images were also obtained.

\section{Imaging findings}

There was overall loss of tissue volume restricted to right side of the face which was not crossing the midline. There was reduction in thickness of the subcutaneous fat on right side of the face. The ipsilateral platysma and buccinators muscle appeared relatively thinned, and the right side of the face appeared sunken. (Fig 2a \& 2b), Nasal septum was deviated to the left with septal spur.

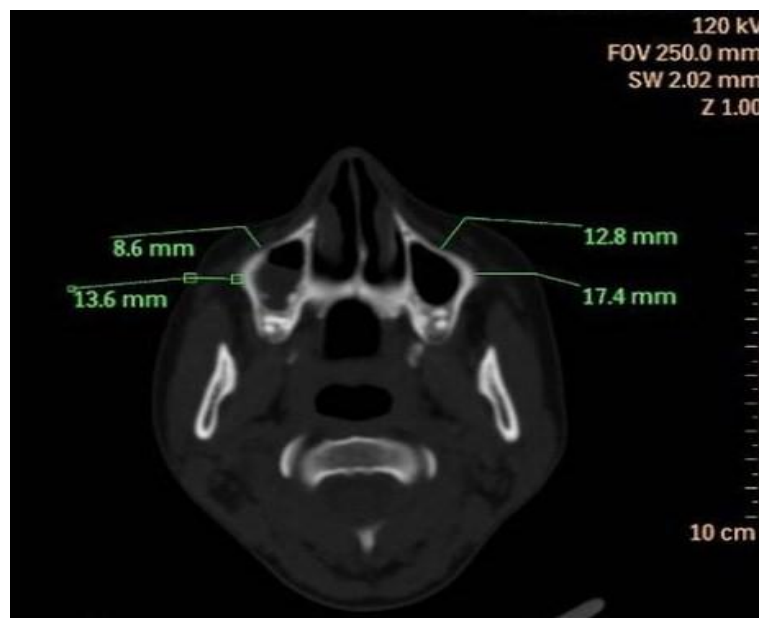

Fig 2a: Axial Bone Window Image at the level of maxillary sinuses shows reduced soft tissue thickness on the right side. However underlying bones and sinus are unaffected in this case.

Eyeballs were symmetrically positioned and no enopthalamus was seen. Mastication muscles, tongue, parotid and submandibular glands and parapharyngeal space and fat planes were bilaterally symmetrical. Bones and paranasal sinuses were bilaterally symmetrical.

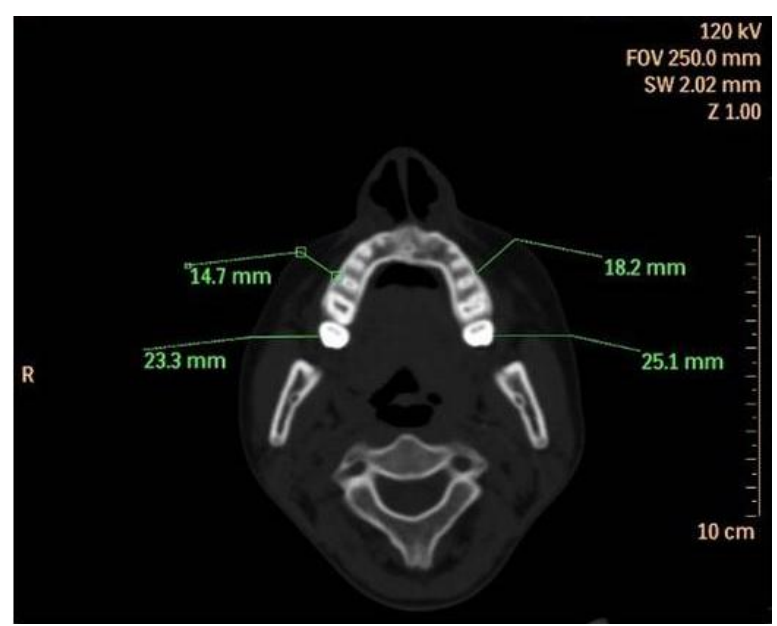

Fig 2b: Axial Bone Window Image at the level of mandible shows reduced soft tissue thickness on the right side. Mandible appears symmetrical.

\section{Table 1: Clinical Features (Approximate Incidence)}

- Facial hemiatrophy of fat, skin, connective tissue, muscle, and/or bone (100\%)

- Hemiatrophy of contralateral or ipsilateral arm, trunk, or leg $(20 \%)$

- Atrophy of tongue (25\%)

- Dental abnormalities (50\%)

- Trismus /jaw symptoms (including hemi-masticatory spasm) (35\%)

- Migraine/facial pain (45\%)

- Ocular abnormalities including globe retraction, uveitis, pupillary abnormalities, restrictive ocular myopathy (mimicking Duane's syndrome), heterochromia

- Epilepsy (10\%), sometimes associated with ipsilateral brain changes on MRI (5\%)

- Vitiligo, hair depigmentation/ hyper- pigmentation (20\%) 


\section{Table 2: Imaging Features}

\section{Radiographs}

- Dental radiograph/orthopantomogram - most commonly unilateral short hypoplastic roots, delayed ipsilateral tooth eruption, missing teeth, oligodontia, microdontia and unilateral thinning of mandible

- Skull radiographaph - may be normal if bones are uninvolved, however may show mandibular thinning, maxillary bone and maxillary sinus may be small in size , ipsilateral TM joint abnormalities

\section{CT}

- Soft tissue- Facial hemiatrophy of fat (most commonly) and muscles, ipsilateral parotid and submandibular gland atrophy \& hemiatrophy of tongue.

- Bone - apparently normal or small, unilateral small maxillary sinus.

- Orbit and eyeballs- small bony orbit and enophthalmus.

\section{MRI}

Usually ipsilateral but sometimes contra-lateral in grey and white matter

- Focal or hemiatrophy,

- Calcifications,

- Cortical dysplasia

- White matter T2 hyperintensity,

- Meningeal thickening, Vascular- aneurysms, and intracranial vascular malformations.

\section{Discussion}

Parry Romberg Syndrome usually present with facial asymmetry and epilepsy with average age of onset around 10 years old with about one third starting after the age of 15. The disease typically progresses over a few years (but sometimes much longer) and then arrests. Disease is more common in females and more commonly seen on the left side. It is probably an autoimmune mediated condition. It needs to be differentiated from congenital hemifacial microsomia. Reports of Parry-Romberg syndrome occuring after head trauma and surgery to the face are known. Possible hereditary etiology is also proposed in few cases. Refer Table 1 for various clinical features. ${ }^{1}$

\section{Role of Imaging}

CT scan is used for defining the severity of asymmetry and to know about the underlying bone involvement that is crucial for planning cosmetic surgery. ${ }^{4}$ CT Head may also show calcific lesions in brain. MRI in conjunction with EEG is used to depict brain abnormalities and possible epileptogenic focus. Refer table 2 for various imaging findings. ${ }^{1,2}$

\section{Treatment}

There is no definitive treatment available. Restorative plastic surgery may be useful to correct cosmetic problems which includes fat or silicon implants, flap/pedicle grafts, or bone implants. $^{2}$

Patients tend to be moderately satisfied with the outcome of these procedures. However, 
they should be made aware that fat injections may be resorbed if the disease is still active.

In progressive stage of the disease, treatments used include methotrexate (for which there is limited evidence in linear scleroderma), corticosteroids, cyclophosphamide, and azathioprine but benefits are not proven definitely. Epilepsy may be steroid responsive. ${ }^{1,2}$

\section{References}

1. Parry-Romberg syndrome, Jone Stone, Practical Neurology 2006;6: 185-188.

2. J Oral Maxillofac Res 2011; 2(2):1.

3. North American NeuroOphthalmology Society, 282, Poster 124, Parry-Romberg Syndrome: Presentation of Two Patients; Review of Neuro Ophthalmologic Manifestations.

4. Human malformation and related anomalies, By Roger E. Stevenson, Judith G. Hall, 8.9 congenital anomalies of facial skeleton. 